BULL. AUSTRAL. MATH. SOC.

VOL. $20(1979), 227-231$.

\title{
A nonlinear complementarity problem for monotone functions
}

\section{Sribatsa Nanda and Ujagar Patel}

\begin{abstract}
In this note we prove that for a monotone function that fixes the origin, the complementarity problem for $c^{n}$ always admits a solution. If, moreover, the function is strictly monotone, then zero is the unique solution. These results are stronger than known results in this direction for two reasons: firstly, there is no condition on the nature of the cone and secondly, no feasibility assumptions are made.
\end{abstract}

\section{Preliminaries}

Let $c^{n}$ denote the $n$-dimensional complex space with hermitian norm and the usual inner product. Let $K$ denote a closed convex cone in $c^{n}$ and $K^{*}$ its polar; that is,

$$
K^{*}=\left\{y \in C^{n}: \operatorname{re}(x, y) \geq 0 \text { for all } x \in K\right\}
$$

Given a continuous function $g: c^{n} \rightarrow c^{n}$, the nonlinear complementarity problem in $C^{n}$ consists of finding a point $z$ such that (1.1) $z \in K, g(z) \in K^{*}$, and $\operatorname{re}(g(z), z)=0$.

In this note we shall be concerned with the solution of the nonlinear complementarity problem for monotone functions. A mapping $f: c^{n} \rightarrow c^{n}$ is said to be monotone on $K$ if $\operatorname{re}(f(x)-f(y), x-y) \geq 0$ for every $(x, y) \in K \times K$, and strictly monotone if strict inequality holds whenever

Received 23 February 1979. Corrected 5 May 1979. 
$x \neq y$. The function $f$ is said to be strongly monotone if there is a constant $c>0$ such that, for each $(x, y) \in K \times K$,

$$
\operatorname{re}(f(x)-f(y), x-y) \geq c\|x-y\|^{2} .
$$

For each real number $r \geq 0$, we denote by $K_{r}$ the following set

$$
K_{r}=\{x \in K:\|x\| \leq \boldsymbol{r}\} \text {. }
$$

Several authors (see references [1], [2], [3], [5], [6], and [7]) have discussed the complementarity problem for $R^{n}$ and $C^{n}$. The result we present here is an improvement of the results contained in the references above for two reasons. Firstly, no assumptions are made on the nature of the cone (such as polyhedral, pointed, and so on). Secondly, we do not make any feasibility assumption.

\section{The main theorem}

We start by mentioning a modified version of a result of Hartman and Stampacchia [4].

LEMMA 2.1. Let $g: K \rightarrow C^{n}$ be a continuous map. Then there is a point $z_{r} \in K_{r}$ such that

$$
\operatorname{re}\left(g\left(z_{p}\right), z-z_{p}\right) \geq 0
$$

for alz $z \in K_{r}$.

Proof. $K_{r}$ is a closed convex and bounded set. Therefore Proposition 2.1 of [6] applies, and the desired result follows.

LEMMA 2.2. Let $g: K \rightarrow C^{n}$ be a continuous monotone function on $K$ that fixes the origin, and let $x \in K$. Then the continuous function $\theta: R^{+} \rightarrow R$ defined by

$$
\theta(r)=\operatorname{re}(g(r x), r x)
$$

is monotone increasing.

Proof. Let $s \in R$. Since $g$ is monotone, $\operatorname{re}(g(s x)-g(0), s x) \geq 0$, implying that $\mathrm{re}(g(s x), s x) \geq 0$. For any $r, s \in R$ with $r<s$, we have

$$
\operatorname{re}(g(r x)-g(s x),(r-s) x) \geq 0 \text {. }
$$


Since $r-s<0$, we have

$$
\operatorname{re}(g(r x)-g(s x), x) \leq 0
$$

Thus

$$
\operatorname{re}(g(r x), r x) \leq \operatorname{re}(g(s x), r x)=(r / s) \operatorname{re}(g(s x), s x) \leq \operatorname{re}(g(s x), s x),
$$

which is the required result.

THEOREM 2.3. Let $g: K \rightarrow C^{n}$ be a continuous function that fixes the origin and which, moreover, is monotone on $K$. Let $z_{r} \in K_{r}$ be the point as obtained in Lemma 2.1. Then $z_{r}$ is a solution of the complementarity problem (1.1).

Proof. Note first of all that since

$$
\operatorname{re}\left(g\left(z_{p}\right), z-z_{p}\right) \geq 0 \text { for all } z \in K_{r},
$$

it follows by taking $z=0$ that

$$
\operatorname{re}\left(g\left(z_{p}\right), z_{p}\right) \leq 0 .
$$

Let $t \in[0,1]=I$. We then have from Lemma 2.2 that

$$
\operatorname{re}\left(g\left(t z_{p}\right), t z_{p}\right) \leq \operatorname{re}\left(g\left(z_{r}\right), z_{p}\right) \leq \operatorname{re}\left(g\left(z_{p}\right), z\right)
$$

for all $z \in K_{r}$. It is also evident that the second inequality above holds for all $z \in K$. Thus we have

$$
\operatorname{re}\left(g\left(z_{p}\right), z\right) \leq-\operatorname{re}\left(g\left(z_{p}\right), z_{p}\right) \leq-\operatorname{re}\left(g\left(t z_{p}\right), t z_{p}\right) .
$$

Since $-\operatorname{re}\left(g\left(z_{p}\right), z_{p}\right) \geq 0$ by virtue of (2.2), it follows that

$$
-r e\left(g\left(t z_{p}\right), t z_{p}\right) \geq 0 \text {, }
$$

so that we can apply Cauchy-Schwartz inequality to get

$$
-\operatorname{re}\left(g\left(z_{p}\right), z\right) \leq-\operatorname{re}\left(g\left(t z_{p}\right), t z_{p}\right) \leq\left\|g\left(t z_{r}\right)\right\|\left\|t z_{r}\right\|
$$

for all $z \in K$ and $t \in I$.

Observe that the set $\left\{t z_{r}: t \in I\right\}$ is compact; thus $g$ attains a maximum on this set, say $M$. Thus from (2.3) we have the inequality

$$
-\operatorname{re}\left(g\left(z_{p}\right), z\right) \leq M\left\|z_{p}\right\|,
$$


which holds for all $z \in K$ and $t \in I$. Since $t$ can be chosen to be arbitrarily small, it follows that

$$
-\mathrm{re}\left(g\left(z_{r}\right), z\right) \leq 0
$$

for all $z \in K$. This shows that $g\left(z_{\gamma}\right) \in K^{*}$, and, moreover, (2.4) together with the inequality (2.2) implies that $\operatorname{re}\left(g\left(z_{r}\right), z_{r}\right)=0$. Thus $z_{p}$ is a solution to the complementarity problem.

THEOREM 2.4. Let $f: K \rightarrow C^{n}$ be a continuous function that fixes the origin and which, moreover, is strictly monotone on $K$; then zero is the unique solution to the complementarity problem.

Proof. That a solution exists is clear from the theorem above. Moreover, due to strict monotonicity, such a solution is unique. Suppose that $z_{0}$ is the solution. Assume to the contrary that $z_{0} \neq 0$. Let $\left\|z_{0}\right\|=k>0$, and let $r=\frac{1}{2} k$. By applying Lemma 2.1 we get a $z_{r} \in K_{r}$ which is the solution of the complementarity problem. Since the solution is unique, $z_{r}=z_{0}$. This is impossible since $\left\|z_{r}\right\| \leq \frac{1}{2} k$. Thus we arrive at a contradiction and $z_{0}$ must be zero.

REMARK 1. A function $g: c^{n}+c^{n}$ is said to be $\alpha$-monotone on $K$ if for some function $\alpha: R^{+} \rightarrow R^{+}$which is monotone increasing such that $\alpha(r) \rightarrow \infty$ as $r \rightarrow \infty$, the following inequality is satisfied:

$$
\operatorname{re}(g(x)-g(y), x-y) \geq \alpha(\|x-y\|)\|x-y\| \text {. }
$$

In case $\alpha(r)=c r$ for some $c>0, g$ is said to be strongly monotone. It is clear that $\alpha$-monotone functions are strictly monotone. Therefore Theorem 2.4 applies and zero is the unique solution to the complementarity problem under the same conditions.

REMARK 2. The arguments of Theorem 2.3 and Theorem 2.4 go through in case we have $R^{n}$ instead of $C^{n}$. 


\section{References}

[1] M.S. Bazaraa, J.J. Goode and M.Z. Nashed, "A nonlinear complementarity problem in mathematical programing in Banach spaces", Proc. Amer. Math. Soc. 35 (1972), 165-170.

[2] B.C. Eaves, "On the basic theorem of complementarity", Math. Programing 1 (1971), 68-75.

[3] G.J. Habetler and A.L. Price, "Existence theory for generalized nonlinear complementarity problems", J. Optim. Theory App Z. 7 (1971), 223-239.

[4] Philip Hartman and Guido Stampacchia, "On some non-linear elliptic differential-functional equations", Acta Math. 115 (1966), $271-310$.

[5] S. Karamardian, "Generalized complementarity problem", J. Optim. Theory App 2. 8 (1971), 161-168.

[6] Sribatsa Nanda and Sudarsan Nanda, "A complex nonlinear. complementarity problem", Bull. Austral. Math. Soc. 19 (1978), $437-444$.

[7] Sribatsa Nanda and Sudarsan Nanda, "On stationary points and the complementarity problem", Bul2. Austral. Math. Soc. 20 (1979), $77-86$.

Department of Mathematics,

Regional Engineering College,

Rourkela,

Orissa,

India;

Department of Mathematics, Gangadhar Meher College,

Sambalpur,

Orissa,

India. 